\title{
Altura de voo de insetos-praga do arroz irrigado
}

\author{
Eduardo Rodrigues Hickel ${ }^{1}$ e Débora Dal Zotto²
}

\begin{abstract}
Resumo - Dentre as pragas do arroz irrigado em Santa Catarina estão a bicheira-da-raiz, Oryzophagus oryzae (Costa Lima) e Helodytes sp. (ambas Coleoptera: Curculionidae), a lagarta-boiadeira, Nymphula spp. e a noiva-do-arroz, Rupela albinella Cramer (ambas Lepidoptera: Pyralidae), o percevejo-do-grão, Oebalus spp. (Hemiptera: Pentatomidae) e o cascudo-preto, Euetheola humilis (Burmeister) (Coleoptera: Scarabaeidae). A dispersão desses insetos no ambiente é ampla, porém se desconhece a sua capacidade de transpor obstáculos verticais na rota de voo. Assim, foi objetivo desta pesquisa verificar a altura de voo dos insetos-praga do arroz irrigado. Duas armadilhas luminosas, modelo "Luiz de Queiroz" foram instaladas em uma lavoura de arroz irrigado em Itajaí, SC, sendo uma delas ao nível superior das plantas e a outra a seis metros de altura do solo. No período de setembro de 2014 a dezembro de 2015, as armadilhas foram ligadas diariamente das 16 às 9 horas, exceto de maio a agosto de 2015, quando permaneceram desligadas. Todas as espécies citadas alcançaram a altura de voo de $6 \mathrm{~m}$ e, portanto, são capazes de ultrapassar obstáculos até essa altura. Contudo, apenas $O$. oryzae e $E$. humilis voam habitualmente por esse estrato.
\end{abstract}

Termos para indexação: ecologia de insetos; dispersão; comportamento; manejo de pragas; Oryza sativa.

\section{Flight height of Brazilian rice insect pests}

\begin{abstract}
The South American rice water weevil, Oryzophagus oryzae (Costa Lima), and the big rice water weevil, Helodytes sp. (both Coleoptera: Curculionidae), the rice caseworm, Nymphula spp. and the white stem borer, Rupela albinella Cramer (both Lepidoptera: Pyralidae), the rice stink bug, Oebalus spp. and the black beetle, Euetheola humilis (Burmeister) (Coleoptera: Scarabaeidae) are among insect pests of irrigated rice in Brazil. The dispersal of these insects is extensive, but their ability to cross vertical obstacles in the flight path is unknown. So, this research aimed to verify the flight height of Brazilian rice insect pests. Two light traps, model "Luiz de Queiroz", was set in an irrigated rice field at Epagri Experimental Station, in Itajaí, SC, one at the top level of plants and the other six meters high. From September 2014 to December 2015 the light traps were turned on daily, from 4pm to 9am, except from May to August of 2015 when they remain turned off. All the pest species reached $6 \mathrm{~m}$ flight height and, therefore, can overcome obstacles to this point. However, only $O$. oryzae and $E$. humilis usually fly by this layer.
\end{abstract}

Index terms: insect ecology; dispersion; behavior; integrated pest management; Oryza sativa.

\section{Introdução}

Os insetos foram os primeiros seres alados a surgir na Terra (GILLOTT, 1995). Isso Ihes possibilitou explorar inúmeros habitats, dificilmente alcançados por seres andantes (GULLAN \& CRANSTON, 2008). O voo também possibilitou aos insetos ampliar sua área de dispersão e colonizar novas terras, transformando-os no grupo mais diversificado de seres vivos do planeta (GILLOTT, 1995; WOOD, 2007).

A altura de voo dos insetos tem sido alvo de diversas pesquisas, quer para fins científicos, quer para o manejo de pragas (WOOD, 2007; BYERS, 2011). No caso do manejo de pragas, a altura de voo subsidia os estudos de posicionamento de armadilhas no campo, visando ao monitoramento ou à coleta massal da espécie-alvo (SU \& WOODS, 2001; MACHADO, 2013; VENTER et al., 2009; BYERS, 2011). Outra aplicação desse conhecimento está relacionada ao alcance do inseto em dispersão ou migração (BRAVERMAN \& LINLEY, 1993; WOOD, 2007) e sua capacidade de transpor barreiras verticais na rota de voo. Isso possibilita estudar a viabilidade do emprego de barreiras físicas como medida de manejo integrado de pragas (GUIMARÃES et al., 1997; ARMANDO, 2002; SHIMBORI et al., 2012).

Os estudos sobre a altura de voo dos insetos normalmente envolvem o uso de algum aparato para a captura dos indivíduos (e.g. uma armadilha) ou então a análise de detecção de eco feita por radar (WOOD, 2007; BYERS, 2011). Para estratos inferiores de voo (até $10 \mathrm{~m}$ de altura) o uso de armadilhas é preponde- rante (BYERS, 2011; MACHADO, 2013). Alguns insetos-praga do arroz irrigado podem ser monitorados com armadiIhas, sendo a armadilha luminosa uma das mais úteis, tendo em vista o fototropismo positivo das espécies (CAMARGO et al., 1990; HAQ et al., 2006; BAO et al., 2008).

Os insetos-praga do arroz com fototropismo positivo incluem os adultos da bicheira-da-raiz, Oryzophagus oryzae (Costa Lima) e Helodytes sp. (ambos Coleoptera: Curculionidae), mariposas da lagarta-boiadeira, Nymphula spp. e da noiva-do-arroz, Rupela albinella Cramer (ambas Lepidoptera: Pyralidae), o percevejo-do-grão, Oebalus spp. (Hemiptera: Pentatomidae) e o cascudo-preto, Euetheola humilis (Burmeister) (Coleoptera: Scarabaeidae). Essas espécies voam durante a noite, porém a altura 
de voo é desconhecida. Isso porque os estudos de monitoramento e flutuação populacional baseiam-se na captura de indivíduos com armadilhas luminosas que operam ao nível da lavoura (HICKEL, 2013; 2014; NISHI, 2015).

Dessa forma, torna-se importante conhecer a altura de voo das pragas do arroz irrigado para saber a capacidade de transporem obstáculos verticais na rota de voo e assim conjecturar sobre a viabilidade do emprego de barreiras físicas verticais como medida de manejo integrado de pragas.

\section{Material e métodos}

O estudo foi conduzido na Estação Experimental da Epagri em Itajaí, Santa Catarina, de setembro de 2014 a dezembro de 2015, numa quadra de arroz irrigado, de 0,15ha $\left(26^{\circ} 56^{\prime} 44^{\prime \prime} \mathrm{S}\right.$ $48^{\circ} 45^{\prime} 42^{\prime \prime} \mathrm{)}$ ). Esta quadra faz limite a norte, sul e leste com outras quadras de arroz e a oeste com o leito seco original do Rio Itajaí Mirim. O cultivar utilizado foi o Epagri 106, que apresenta ciclo precoce. Na safra 2014/15 a semeadura ocorreu em 28/08/2014, na densidade de $100 \mathrm{~kg} . \mathrm{ha}^{-1}$. Na safra 2015/16, em função da persistência de baixas temperaturas e alta precipitação, optou-se pelo transplante mecânico de mudas, em 29/09/2015, no espaçamento de 15 $x 20 \mathrm{~cm}$. O sistema de cultivo adotado foi o pré-germinado, conforme preconizado por Eberhardt \& Schiocchet (2015), porém sem a aplicação de inseticidas para controle da bicheira-da-raiz.

Em agosto de 2014, duas armadilhas luminosas, modelo "Luiz de Queiroz" equipadas com luz negra (T8 15W BL $\mathrm{LE})$, foram instaladas em postes de concreto, uma delas na altura ${ }^{3}$ do nível superior das plantas ( $1 \mathrm{~m}$ do solo) e a outra a $6 \mathrm{~m}$ de altura. A altura de $6 \mathrm{~m}$ foi definida em função da logística necessária para instalação e operação da armadiIha luminosa e por essa altura se aproximar da altura normal dos fragmentos florestais existentes entre as propriedades rurais da região.

A armadilha ao nível das plantas foi posicionada ao lado da taipa, a meia distância do maior comprimento da quadra e a outra (a $6 \mathrm{~m})$, no dique de contenção de cheias, no canto nordeste da quadra. Essa armadilha era erguida e baixada com auxílio de um cabo de aço e roldanas presas ao poste. Para limitar a captura de insetos maiores, uma tela de náilon ( 5 × 5mm de malha) foi colocada circundando as aletas das armadilhas "Luiz de Queiroz".

No período de 04 de setembro de 2014 a 17 de dezembro de 2015, as armadilhas foram ligadas diariamente, das 16 às 9 horas, exceto nos meses de maio a agosto de 2015, quando permaneceram desligadas, devido à baixa captura de insetos-praga do arroz irrigado (HICKEL, 2013, 2014). Os insetos atraídos foram aprisionados em sacos plásticos de 20L, fixados no funil coletor das armadilhas, de onde posteriormente efetuou-se a triagem dos insetos-praga do arroz e a contagem dos indivíduos em laboratório. Com o registro das contagens foram confeccionados os gráficos de flutuação populacional para cada estrato de altura. Os valores acumulados de coleta semanal de cada espécie foram transformados em $(x+0,5)^{0,5}$, sendo a significância do teste $t$ obtida em planilha eletrônica Excel, executando-se a rotina "teste.t".

\section{Resultados e discussão}

Todas as espécies estudadas alcançaram a altura de voo de $6 \mathrm{~m}$ e, portan- to, podem ultrapassar obstáculos até essa altura (Tabela 1). Contudo, apenas os adultos de $O$. oryzae e $E$. humilis voaram habitualmente na altura de $6 \mathrm{~m}$, pois não houve diferença para as capturas ao nível das plantas (1m). As espécies Helodytes sp., Nymphula spp., $R$. albinella e Oebalus spp. chegaram aos $6 \mathrm{~m}$ provavelmente em resposta ao estímulo atrativo da luz UV da armadiIha luminosa. Isso, embora resulte de alteração do comportamento normal da espécie, conforme argumenta Byers (2011), comprova que, por estímulos externos, os indivíduos dessas espécies conseguem eventualmente ultrapassar obstáculos verticais naturais e se dispersar para novas áreas.

Na compilação efetuada por Byers (2011), a altura média de voo de diversas espécies de insetos situou-se na faixa de 0,17 a 5,40m, sendo que as espécies que voam mais alto têm, via de regra, maior amplitude de estrato de voo ${ }^{4}$. Seguindo essa tendência, $O$. oryzae e $E$. humilis devem ter amplitude de estrato de voo superior às demais espécies estudadas, visto terem sido frequentemente capturadas aos $6 \mathrm{~m}$ de altura.

Byers (2011) também argumenta que o espalhamento dos indivíduos de uma espécie no estrato de voo segue distribuição normal, ou seja, há uma altura média preferida e um desvio padrão em relação a essa média, que define a faixa ótima de voo. Assumindo válida essa hipótese para $O$. oryzae e $E$.

Tabela 1. Total de indivíduos capturados por espécie e altura de voo de insetos-praga do arroz irrigado. Itajaí, SC, setembro de 2014 a dezembro de 2015

Table 1. Total number of trapped individuals per species and flight height of irrigated rice pests. Itajai, SC, September 2014 to December 2015

\begin{tabular}{lccc}
\hline \multirow{2}{*}{ Espécie } & \multicolumn{2}{c}{ Altura de voo } & \multirow{2}{*}{ Probabilidade $^{1}$} \\
\cline { 2 - 3 } & $1 \mathrm{~m}$ & $6 \mathrm{~m}$ & \\
\hline Oryzophagus oryzae & 18.857 & 17.853 & 0,202 \\
Helodytes sp. & 1.003 & 299 & 0,001 \\
\hline Nymphula spp. & 3.062 & 507 & $<0,001$ \\
Rupela albinella & 67 & 32 & 0,015 \\
Oebalus spp. & 3.313 & 209 & 0,024 \\
\hline Euetheola humilis & 385 & 332 & 0,517 \\
\hline
\end{tabular}

1/ Probabilidade do teste t para altura de voo, a 5\% de significância, aplicado para as séries de coleta semanal $(n=40)$.

\footnotetext{
${ }^{3}$ Altura entre o nível do solo e a abertura do funil coletor da armadilha luminosa.

${ }^{4}$ Entende-se por estrato de voo o espaço delimitado pelos limites inferior e superior de altura no qual os indivíduos de uma espécie voam.
} 
humilis, verifica-se que essas espécies podem voar bem acima dos $6 \mathrm{~m}$ de altura, pois as coletas a $1 \mathrm{~m}$ e a $6 \mathrm{~m}$ foram similares (Tabela 1). Assim, a limitação na altura de voo estaria sendo consequência da máxima altura de instalação da armadilha. Flechtmann et al. (1997), reavaliando a altura de voo de besouros bostriquídeos, concluíram que esses insetos voam além da máxima altura estabelecida nos estudos anteriores.

Outra hipótese seria $O$. oryzae e $E$. humilis não terem altura preferida de voo, conforme constatado para algumas espécies de besouros escolitídeos e bostriquídeos (FLECHTMANN et al., 1997; ABREU et al., 2001; PERES FILHO et al., 2012). Isto resultaria na exploração por igual de todo o estrato de voo pelos indivíduos.

Visto que a altura de voo se correlaciona com a amplitude e a rapidez da dispersão de dado inseto (BRAVERMAN \& LINLEY, 1993; WOOD, 2007), supõese que $O$. oryzae e $E$. humilis têm maior poder dispersivo nas áreas produtoras de arroz irrigado. Isso é corroborado pelas infestações epidêmicas de $O$. oryzae, bem como pela distância em que se verificam indivíduos em áreas remotas (HICKEL, 2013). E. humilis também é bom voador, alcançando grandes distâncias em dispersão (ALZUGARAY, 2008; BILLEISEN \& BRANDENBURG, 2014). Por essa argumentação, esperase que Helodytes sp., Nymphula spp., $R$. albinella e Oebalus spp. se disper- sem menos pelas áreas de cultivo. Os percevejos Oebalus spp. têm, reconhecidamente, limitada dispersão por voo, ficando esses movimentos restritos na faixa de 50 a $250 \mathrm{~m}$ (FERREIRA et al., 2001). As mariposas Nymphula spp. e $R$. albinella também têm dispersão limitada nas lavouras de arroz irrigado, sendo estimado para Nymphula spp. o alcance máximo de $1 \mathrm{~km}$ (REISSIG et al., 1986).

Verifica-se para $O$. oryzae e E. humilis que as flutuações populacionais foram equivalentes nas duas alturas de coleta (Figuras 1 e 2). As coletas obtidas aos $6 \mathrm{~m}$ para Helodytes sp., Nymphula spp., $R$. albinella e Oebalus sp. foram menos intensas e ocorreram quando as populações estavam altas ao nível das plantas (Figuras 1, 2 e 3). Nesse caso, o maior número de indivíduos no estrato inferior aparentemente aumentou a probabilidade de alguns deles responderem ao estímulo atrativo da lâmpada UV aos $6 \mathrm{~m}$ de altura.

Milanez et al. (2001), Milanez \& Chiaradia (2005) e Chiaradia et al. (2008) obtiveram resultados similares para Diabrotica speciosa (Germar) (Coleoptera: Chrysomelidae), Astylus variegatus (Germar) (Coleoptera: Melyridae) e Diaphorina citril Kuwa. (Hemiptera: Psyllidae) respectivamente. Esses autores verificaram que os insetos ocorriam ao longo do tempo em todas as alturas pré-estabelecidas, porém sempre em maior número nas respectivas alturas preferidas de voo.
A faixa ótima de voo é preponderante para o estabelecimento da altura de instalação de armadilhas atrativas, conforme constatado para mariposas Tortricidae, besouros Nitidulidae e Scolytidae e cigarrinhas Circulifer tenellus (Baker) (TOMLINSON, 1970; MEYERDIRK \& OLDFIELD, 1985; BARTELT et al., 1994; MACHADO, 2013). Neste aspecto, a altura tradicional de instalação de armadilhas luminosas em lavouras de arroz irrigado, onde a abertura do funil coletor fica no nível superior das plantas (cerca de $1 \mathrm{~m}$ do solo), está bem ajustada à altura de voo dos insetos-praga do cultivo.

Tendo em vista a altura de voo alcançada pelos insetos-praga do arroz irrigado, torna-se inviável a prospecção de barreiras verticais (e.g. quebra-ventos), como medida de manejo de pragas, visando conter a dispersão desses insetos para as áreas de lavoura. $\mathrm{O}$ uso de barreiras verticais normalmente é pesquisado para insetos de dimensões reduzidas e de pouca atividade de voo, como tripes, pulgões, psilídeos ou outros hemípteros diminutos (GUIMARÃES et al., 1997; SHIMBORI et al., 2012). No caso dos insetos-praga do arroz irrigado, a atividade de voo é intensa e frequente durante todo o período que estes permanecem infestando as lavouras.

\section{Conclusão}

As espécies $O$. oryzae, Helodytes sp., Nymphula spp., R. albinella, Oebalus
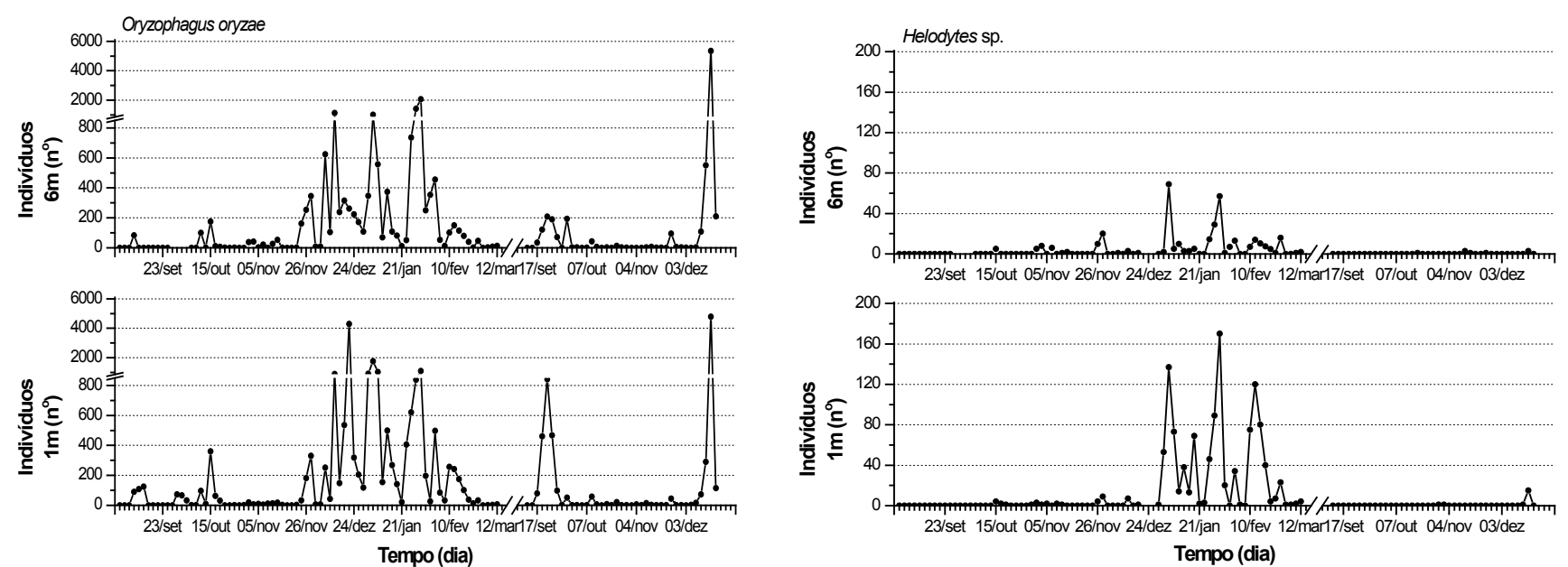

Figura 1. Flutuação populacional de adultos da bicheira-da-raiz (Oryzophagus oryzae e Helodytes sp.), de acordo com a altura de voo. Itajaí, SC, setembro de 2014 a dezembro de 2015

Figure 1. Population dynamics of rice water weevil adults (Oryzophagus oryzae and Helodytes sp.), according to flight height. Itajai, SC, September 2014 to December 2015 

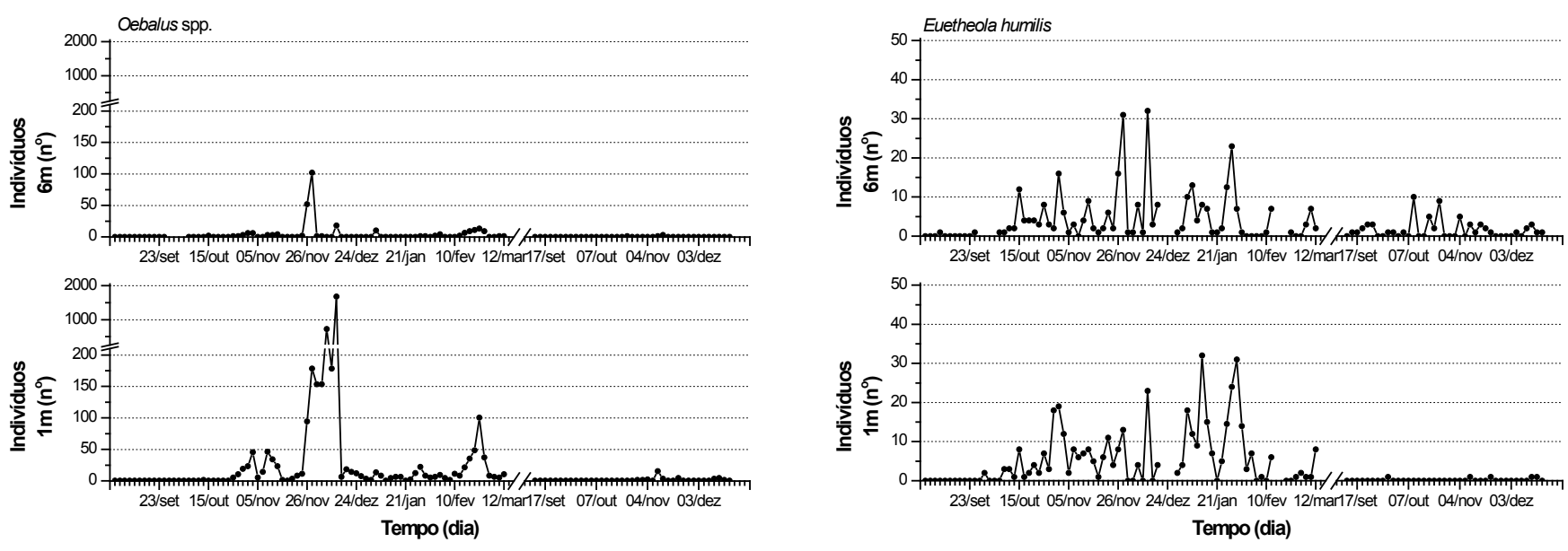

Figura 2. Flutuação populacional de percevejos-do-grão (Oebalus spp.) e do cascudo-preto (Eutheola humilis), de acordo com a altura de voo. Itajaí, SC, setembro de 2014 a dezembro de 2015

Figure 2. Population dynamics of rice stink bugs (Oebalus spp.) and black beetle (Eutheola humilis), according to flight height. Itajai, SC, September 2014 to December 2015
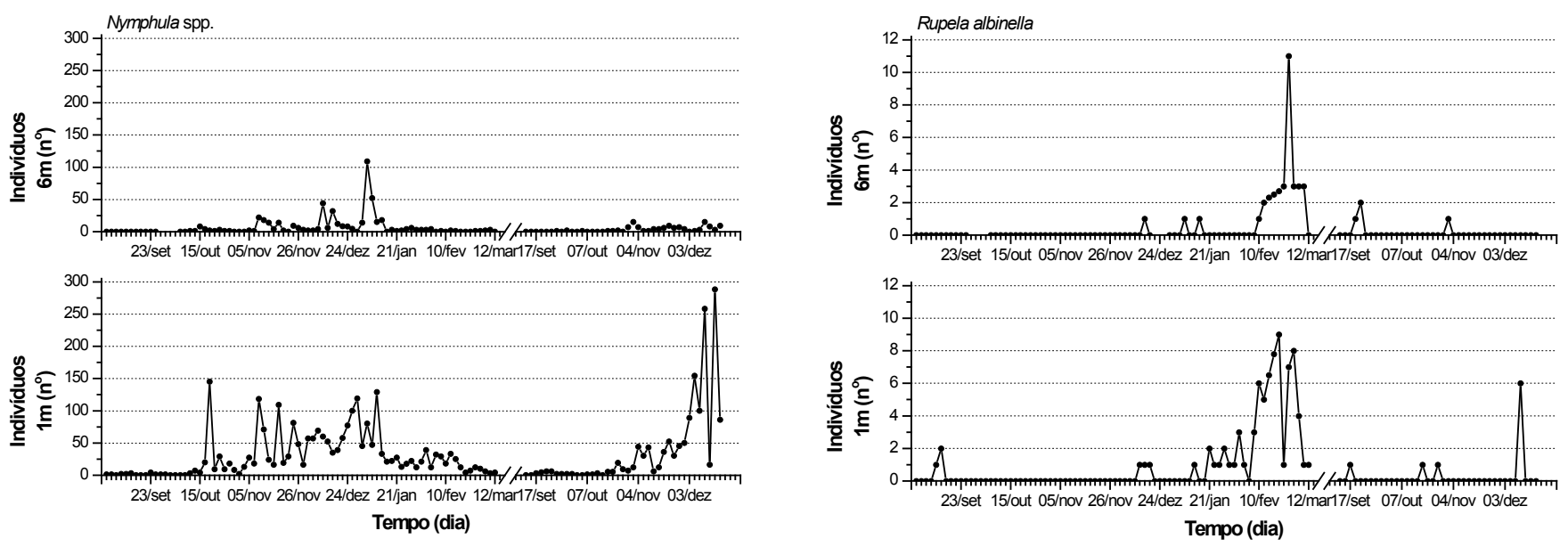

Figura 3. Flutuação populacional de mariposas da lagarta-boiadeira (Nymphula spp.) e da noiva-do-arroz (Rupela albinella), de acordo com a altura de voo. Itajaí, SC, setembro de 2014 a dezembro de 2015

Figure 3. Population dynamics of rice caseworm moths (Nymphula spp.) and the white stem borer moths (Rupela albinella), according to flight height. Itajaí, SC, September 2014 to December 2015

spp. e E. humilis alcançam a altura de voo de $6 \mathrm{~m}$ e, portanto, podem ultrapassar obstáculos até essa altura.

$\mathrm{O}$ gorgulho aquático 0 . oryzae e o cascudo-preto $E$. humilis voam habitualmente no estrato de 1 a $6 \mathrm{~m}$ de altura.

\section{Agradecimentos}

À Fundação de Amparo à Pesquisa e Inovação do Estado de Santa Catarina - Fapesc e ao Conselho Nacional de Desenvolvimento Científico e Tecnológico-CNPq, Edital Repensa, pelo suporte financeiro ao desenvolvimento da pesquisa.

\section{Literatura citada}

ABREU, R.L.S.; FONSECA, C.R.V.; HURTADO GUERRERO, J.C.; PAULA, E.V.C.M. Preferência de voo de nove espécies da família Scolytidae (Insecta: Coleoptera) na Amazônia Central. Acta Amazonica, v.31, n.1, p.61-68, 2001. DOI: https://doi.org/10.1590/180943922001311068.

ALZUGARAY, R. El Cascarudo Negro - Quién es, cómo vive, de dónde viene. Revista Arroz, v.15, n.55, p.44-48, 2008.

ARMANDO, M.S. Agrodiversidade: ferramenta para uma agricultura sustentável. Brasília: Embrapa Recursos Genéticos e Biotecnologia, 2002. 23p. (Embrapa Recursos Genéticos e Biotecnologia. Documentos, 75).
Disponível em: https://www.infoteca.cnptia.embrapa.br/bitstream/doc/184360/1/ doc075.pdf. Acesso em: 8 jun. 2016

BAO, L.; BENTANCOURT, C.; PÉREZ, O. Avances en las evaluaciones del gorgojo acuático del arroz (Oryzophagus oryzae) en Uruguay. Revista Arroz, v.15, n.56, p.22-25, 2008.

BARTELT, R.J; VETTER, R.S.; CARLSON, D.G.; BAKER, T.C. Influence of pheromone dose, trap height, and septum age on effectiveness of pheromones for Carpophilus mutilatus and C. hemipterus (Coleoptera: Nitidulidae) in a California date garden. Journal of Economic Entomology, v.87, n.3, p.667675 , 1994. DOI: https://doi.org/10.1093/ jee/87.3.667.

BILLEISEN, T.L. \& BRANDENBURG, R.L. Bi- 
ology and management of the sugarcane beetle (Coleoptera: Scarabaeidae) in turfgrass. Journal of Integrated Pest Management, v.5, n.4, p.1-5, 2014. DOI: https://doi. org/10.1603/IPM14008.

BRAVERMAN, Y.; LINLEY, J.R. Effect of light trap height on catch of culicoides (Diptera: Ceratopogonidae) in Israel. Journal of Medical Entomology, v.30, n.6, p.1060-1063, 1993. DOI: https://doi.org/10.1093/jmedent/30.6.1060

BYERS, J.A. Analysis of vertical distributions and effective flight layers of insects: threedimensional simulation of flying insects and catch at trap heights. Environmental Entomology, v.40, n.5, p.1210-1222, 2011. DOI: https://doi.org/10.1603/EN11043.

CAMARGO, L.M.P.C.A.; LEITE, N.; VELLELA, O.V.; LEITE, L.G.; ASAYAMA, T. Gorgulhos aquáticos (Coleoptera: Curculionidae) que ocorrem em cultivos de arroz irrigado do Vale do Paraíba, SP. Arquivos do Instituto Biológico, v.57, n.1/2, p.51-55, 1990.

CHIARADIA, L.A.; MILANEZ, J.M.; SMANIOTTO, M.A.; DAVILA, M.R.F. Flutuação populacional e altura de captura de Diaphorina citri em pomar de citros. Revista de Ciências Agroveterinárias, v.7, n.2, p.157-159, 2008. Disponível em: http://www.periodicos. udesc.br/index.php/agroveterinaria/article/ download/5340/3546. Acesso em: 15 jun. 2016.

EBERHARDT, D.S.; SCHIOCCHET, M.A. Recomendações para a produção de arroz irrigado em Santa Catarina (sistema pré-germinado). Florianópolis: Epagri, 2015. 92p. (Epagri. Sistema de Produção, 48).

FERREIRA, E.; BARRIGOSSI, J.A.F.; VIEIRA, N.R.A. Percevejos das panículas do arroz: fauna heteroptera associada ao arroz. Santo Antônio de Goias: Embrapa Arroz e Feijão, 2001. (Embrapa Arroz e Feijão. Circular Técnica Online, 43). Disponível em: http:// www.cnpaf.embrapa.br/publicacao/circulartecnica/ct_43/index.htm. Acesso em: 15 jun. 2016.

FLECHTMANN, C.A.H.; GASPARETO, C.L.; TEIXEIRA, E.P. Altura de voo de Bostrichidae (Coleoptera) em Pinus caribea v. hondurensis em Agudos, SP. Revista do Instituto Florestal, v.9, n.1, p.19-26, 1997. Disponível em: https://www.researchgate.net/profile/Carlos_Flechtmann/publication/233416091_ Altura_de_voo_de_Bostrichidae_em_Pinus_caribaea_v_hondurensis_em_Agudos_ SP/links/09e4150a683b519859000000.pdf. Acesso em: 8 jun. 2016.

GILLOTT, C. Entomology. 2 ed. New York: Plenum Press, 1995. 798p.

GULLAN, P.J.; CRANSTON, P.S. Os insetos: um resumo de entomologia. 3 ed. São Paulo:
Rocca, 2008. 440p.

GUIMARÃES, A.M.; PAVAN, M.A.; KUROZAWA, C. Efeito da barreira física com plantas de milho na incidência de vira-cabeça na cultura do tomateiro. Fitopatologia Brasileira, v.22, n.2, p.142-147, 1997.

HAQ, M.; MOZADDEDUL HAQUE, N.M.; REZAUL KARIM, A.N.M. Incidence pattern of rice caseworm (Nymphula sp.). Journal of Agriculture and Rural Development, v.4, n.1-2, p.75-81, 2006. Disponível em: https:// www.banglajol.info/index.php/JARD/article/view/771. Acesso em: 15 jun. 2016.

HICKEL, E.R. Flutuação populacional de adultos da bicheira-da-raiz, Oryzophagus oryzae, e de outras espécies de gorgulhos aquáticos em arroz irrigado. Revista de Ciências Agroveterinárias, v.12, n.3, p.247-254, 2013. Disponível em: http://www.revistas. udesc.br/index.php/agroveterinaria/article/ view/5220. Acesso em: 8 jun. 2016.

HICKEL, E.R. Flutuação populacional de mariposas da lagarta-boiadeira, Nymphula spp., em lavoura de arroz irrigado. Agropecuária Catarinense, v.27, n.3, p.74-77, 2014. Disponível em: http://publicacoes.epagri.sc.gov. $\mathrm{br} /$ index.php/RAC/article/view/563. Acesso em: 15 abr. 2020.

MACHADO, L.M. Determinação da altura de voo de escolitídeos em mata nativa e em povoamento de Pinus taeda. 2013. 57f. Dissertação (Mestrado em Engenharia Florestal) - Universidade Federal de Santa Maria, Santa Maria, 2013. Disponível em: https:// repositorio.ufsm.br/handle/1/8709. Acesso em: 8 jun. 2016.

MEYERDIRK, D.E.; OLDFIELD, G.N. Evaluation of trap color and height placement for monitoring Circulifer tenellus (Baker) (Homoptera: Cicadellidae). The Canadian Entomologist, v.117, n.4, p.505-511, 1985. DOI: https://doi.org/10.4039/Ent117505-4.

MILANEZ, J.M.; CHIARADIA, L.A. Altura de captura e flutuação populacional de adultos de Astylus variegatus (Coleoptera: Melyridae) em citros. In: REUNIÃO SUL-BRASILEIRA SOBRE PRAGAS DE SOLO, 9., 2005, Balneário Camboriú. Anais e Ata... Itajaí: Epagri/EEI, 2005. p.155-156.

MILANEZ, J.M.; CORTINA, J.V.; LAJÚS, C.R.; MENEGUZZI, C.R.; CHIARADIA, L.A. Estudos da altura de voo e flutuação populacional de Diabrotica speciosa (Coleoptera: Chrysomelidae). In: REUNIÃO SUL-BRASILEIRA DE PRAGAS DE SOLO, 8., 2001, Londrina. Anais... Londrina: Embrapa Soja, 2001. p.253-254.

NISHI, Y.P.Y. Manual de cultivo de arroz irrigado en la colonia San Juan. Santa Cruz de la Sierra: Caisy, 2015. 144p.

PERES FILHO, O.; BARBOSA, J.I.; SOUZA,
M.D.; DORVAL, A. Altura de voo de bostriquídeos (Coleoptera: Bostrichidae) coletados em Floresta Tropical Semidecídua, Mato Grosso. Pesquisa Florestal Brasileira, v.32, n.69, p.101-107, 2012. Disponível em: https://www.researchgate.net/profile/Otavio_Peres-Filho/publication/267726324_Altura_de_voo_de_bostriquideos_Coleoptera_Bostrichidae_coletados_em_Floresta_Tropical_Semidecidua_Mato_Grosso/ links/54748fb80cf2778985abe7e8.pdf. Acesso em: 15 jun. 2016.

REISSIG, W.H.; HEINRICHS, E.A.; LITSINGER, J.A.; MOODY, K.; FIEDLER, L.; MEW, T.W.; BARRION, A.T. Illustrated guide to integrated pest management in rice in tropical Asia. Manila: IRRI, 1985. 411p. Disponível em: https://www.researchgate.net/publication/38980487_Illustrated_Guide_to_Integrated_Pest_Management_in_Rice_in_Tropical_Asia. Acesso em: 15 abr. 2020.

SHIMBORI, E.M.; SILVA, F.M.; MOTTA, I.S.; OLIVEIRA, H.N. Avaliação preliminar da influência de barreira vegetal com sansão-docampo (Mimosa caesalpiniaefolia Benth.) sobre a entomofauna em cultura de café (Coffea arabica) orgânico na região de Ivinhema, MS. Cadernos de Agroecologia, v.7, n.2, p.1-5, 2012. Disponível em: https:// www.infoteca.cnptia.embrapa.br/bitstream/doc/938176/1/023shimboriavaliacao. pdf. Acesso em: 8 jun. 2016.

SU, J.C. \& WOODS, S.A. Importance of sampling along a vertical gradient to compare the insect fauna in managed forests. Environmental Entomology, v.30, n.2, p.400-408, 2001. DOI: https://doi.org/10.1603/0046225X-30.2.400.

TOMLINSON, W.E.Jr. Effect of blacklight trap height on catches of moths of three cranberry insects. Journal of Economic Entomology, v.63, n.5, p.1678-1679, 1970. DOI : https:// doi.org/10.1093/jee/63.5.1678.

VENTER, G.J.; HERMANIDES, K.G.; BOIKANYO, S.N.B.; MAJATLADI, D.M.; MOREY, L. The effect of light trap height on the numbers of culicoides midges collected under field conditions in South Africa. Veterinary Parasitology, v.166, n.3-4, p.343-345, 2009. DOI: https://doi.org/10.1016/j.vetpar.2009.09.003.

WOOD, C.R. The biometeorology of highaltitude insect layers. 2007. 223f. Tese (Philosophy Doctor) - University of Reading, Reading, 2007. Disponível em: https:// www.researchgate.net/profile/Curtis_ Wood/publication/41571029_The_biometeorology_of_high-altitude_insect_layers/ links/09e4150ffbcb3a2dc9000000/The-biometeorology-of-high-altitude-insect-layers. pdf. Acesso em: 15 jun. 2016. 